\title{
Stiffness heterogeneity-induced double-edged sword behaviors of carcinoma-associated fibroblasts in antitumor therapy
}

\author{
Jiantao Feng ${ }^{1}$, Shivani Sharma ${ }^{2,3,4}$, Elizabeth $\mathrm{Rao}^{2}$, Xiang $\mathrm{Li}^{5}$, Qiang Zhang ${ }^{5}$, Fulong Liao ${ }^{5,6}$, Jie $\mathrm{He}^{1^{*}}$, \\ Dong $\operatorname{Han}^{5 *}$ and Jianyu Rao ${ }^{2,3,4^{*}}$
}

\begin{abstract}
Carcinoma-associated fibroblasts (CAFs) function as a double-edged sword in tumor progression. However, factors affecting the transition between tumor promotion and inhibition remain to be investigated. Here, we found that the transition was determined by stiffness heterogeneity of the tumor stroma in which tumor cells and CAFs were grown. When tumor cells were grown on a rigid plastic substrate, supernatants from CAFs inhibited the cytotoxic effects of 5fluorouracil. In contrast, when tumor cells were grown on a soft substrate $(5.3 \mathrm{kPa})$, supernatants from CAFs grown on a soft substrate increased the cytotoxicity of 5-fluorouracil. The diverse effects of CAFs were mediated by mechanotransduction factors, including stroma stiffness-induced cytokine expression in CAFs and signal transduction associated with stress fiber formation of CAFs. Moreover, we found that the cytokine expression in CAFs was regulated by nuclear Yesassociated protein, which changed according to cell stiffness, as characterized by atomic force microscopy. Overall, these findings suggested that modulating the mechanotransduction of the stroma together with CAFs might be important for increasing the efficacy of chemotherapy.
\end{abstract}

Keywords: stiffness, carcinoma-associated fibroblast, tumor microenvironment, chemotherapy, atomic force microscopy

\section{INTRODUCTION}

Tumor tissues exhibit increased stiffness compared with normal tissues. Recent evidence has demonstrated that stroma stiffness plays important roles in the resistance of tumors to chemotherapy $[1,2]$. Within stiff tissues, the distribution of stroma stiffness is often heterogeneous in different regions of the invasive tumor, in which geographically distinct regions display different constituents and architectures of the extracellular matrix $[3,4]$. As an integral component of the tumor microenvironment, the extracellular matrix is mainly remodeled by carcinomaassociated fibroblasts (CAFs) [5], which are activated by the altered matrix stiffness and solid stress and function to regulate tumor behaviors [6-8]. Increased crosslinking or assembly of extracellular matrix proteins leads to stiffening of the tumor stroma $[9,10]$, which in turn enhances tumor progression by regulating the motility, proliferation, and even chemotherapeutic resistance of cancer cells $[9,11-13]$. In our previous studies, we also demonstrated that mechanotypical factors alone can directly influence the effects of anticancer drugs, such as cisplatin, taxol, and lapatinib [14,15].

In addition to biomechanical factors, biochemical factors and nonmalignant cells, such as CAFs, immune cells, and endothelial cells in tumor microenvironment, can all affect therapeutic responses [16-18]. CAFs constitute a majority of the structural scaffold of solid tumors and play a critical role in tumor survival and progression via secretion of various growth factors, cytokines, and chemokines and degradation of extracellular matrix proteins [19]. However, CAFs are a double-edged sword whose effect on tumor is very inconsistent [20]. Some studies have shown that CAFs act as regulators to promote tu-

\footnotetext{
${ }^{1}$ Cancer Hospital, Chinese Academy of Medical Sciences, Beijing 100021, China

${ }^{2}$ Department of Pathology and Laboratory Medicine, University of California, Los Angeles, CA 90095, USA

${ }^{3}$ California NanoSystems Institute, University of California, Los Angeles, CA 90095, USA

${ }^{4}$ Jonhson Comprehensive Cancer Institute, University of California, Los Angeles, CA 90095, USA

${ }^{5}$ National Center for Nanoscience and Technology, Beijing 100190, China

${ }^{6}$ Institute of Chinese Materia Medica, China Academy of Chinese Medical Sciences, Beijing 100700, China

* Corresponding authors (emails: JRao@mednet.ucla.edu (Rao J); dhan@nanoctr.cn (Han D); hejie@cicams.ac.cn (He J))
} 
morigenesis, metastasis, and resistance to anticancer drugs [6], whereas other studies have demonstrated that CAFs act as inhibitory regulators of cancer progression, suppressing tumor proliferation and invasion [21]. These apparently opposite effects of CAFs have suggested that mechanotypical factors, such as differing stroma stiffness, may affect the mechanotransduction in CAFs, thereby modulating the effects of CAFs on tumor growth and phenotype. However, the specific mechanisms mediating these effects are still unknown.

Accordingly, in this study, we evaluated the effects of substrate stiffness and CCD-1095Sk human breast fibroblasts on the phenotypes of SK-BR-3 breast cancer cells. Our findings provide important insights into the effects of stroma stiffness heterogeneity on the mechanotransduction of CAFs and the role of CAFs as a doubleedged sword in antitumor therapy.

\section{MATERIALS AND METHODS}

\section{Substrate preparation}

Polydimethylsiloxane (PDMS) samples of different stiffnesses were prepared by modifying the ratio of the 'base' and 'curing agent' of SYLGARD 184 (Dow Corning) as follows: 60:1 (w/w; $46.7 \mathrm{kPa})$ and 80:1 (w/w; $5.3 \mathrm{kPa})$ [14]. After exposure to ultraviolet light at $365 \mathrm{~nm}$ for at least $5 \mathrm{~h}$, the PDMS substrates were incubated with type I collagen (Gibco) at a concentration of $0.1 \mathrm{mg} \mathrm{mL}^{-1}$ for $24 \mathrm{~h}$ at $37^{\circ} \mathrm{C}$. After washing with phosphate-buffered saline (PBS) twice, the substrates were soaked in the cell culture medium at $37^{\circ} \mathrm{C}$ for at least $30 \mathrm{~min}$ prior to the addition of cells.

\section{Cell culture and preconditioned medium (PCM) preparation}

SK-BR-3 breast cancer cells (HTB-30; American Type Culture Collection [ATCC]) were cultured in RPMI1640 medium supplemented with $10 \%$ fetal bovine serum (FBS), $100 \mathrm{U} \mathrm{mL}^{-1}$ penicillin, and $100 \mathrm{mg} \mathrm{mL}^{-1}$ streptomycin (all from Hyclone) in a humidified incubator at $37^{\circ} \mathrm{C}$ (Sanyo) with 5\% $\mathrm{CO}_{2}$. CCD-1095Sk human fibroblasts (CRL-2122; ATCC) were maintained in MEM medium (Gibco) supplemented with $10 \%$ FBS, $100 \mathrm{U} \mathrm{mL}^{-1}$ penicillin, $100 \mathrm{mg} \mathrm{mL}^{-1}$ streptomycin (all from Hyclone), $1.5 \mathrm{~g} \mathrm{~L}^{-1}$ sodium bicarbonate, and $0.11 \mathrm{~g} \mathrm{~L}^{-1}$ sodium pyruvate.

CCD-1095Sk cells were allowed to grow on the substrates with different stiffnesses until reaching $90 \%$ confluence, and only cells from passages 2-9 were used. PCM was prepared by filtering FBS-free RPMI1640 medium or medium containing inhibitors, which had been added to the culture dishes of CCD-1095Sk cells $48 \mathrm{~h}$ earlier. Finally, PCMs from different groups were normalized with fresh medium according to the cell number, which was determined using a cell-counting chamber at least three times per sample.

\section{Proliferation and cytotoxicity analysis}

SK-BR-3 cells were seeded into 96-well culture plates coated with PDMS substrates of different stiffnesses at $1 \times$ $10^{4}$ cells/well and cultured for $24 \mathrm{~h}$. For proliferation analysis, a mixture of PCM and RPMI1640 medium (1:1, $v / v$ ) was used as the culture medium, and cell viability was determined once every $24 \mathrm{~h}$. For cytotoxicity analysis, a mixture of PCM and fresh medium dissolved with 5fluorouracil (5-FU) was added into the culture plate, and the cells were incubated for $48 \mathrm{~h}$ (5-FU). The resulting concentrations of 5 -FU were $0.004,0.008,0.04,0.4$, and $4 \mathrm{mg} \mathrm{mL}^{-1}$.

Culture medium containing CCK-8 (Dojindo; 10:1, v/v) was applied as a working solution for cell viability testing. For each test, the supernatant was removed, and $100 \mu \mathrm{L}$ CCK-8 working solution was added to each well; samples were incubated at $37^{\circ} \mathrm{C}$ for $3 \mathrm{~h}$, and the absorbance at $450 \mathrm{~nm}$ (with reference wavelength of $650 \mathrm{~nm}$ ) was then determined using a multimode plate reader (Enspire, PerkinElmer). The half-maximal inhibitory concentration $\left(\mathrm{IC}_{50}\right)$ was calculated by performing polynominal fit of the survival rate with OriginPro 2016.

\section{Immunostaining and imaging}

After growing on substrates of different stiffnesses for 4 days, CCD-1095Sk cells were washed with PBS and fixed with $4 \%$ paraformaldehyde at $25^{\circ} \mathrm{C}$ for $30 \mathrm{~min}$. The cells were permeabilized for $15 \mathrm{~min}$ with $0.25 \%$ Triton-X in PBS on ice, and nonspecific binding was blocked by incubating with $2 \%$ bovine serum albumin for $1 \mathrm{~h}$ at $37^{\circ} \mathrm{C}$. The samples were then incubated at $4^{\circ} \mathrm{C}$ overnight with the primary antibody as indicated (anti- $\alpha-S M A, 1: 100$, SAB2500963 [Sigma-Aldrich]; anti-FAP, 1:100, sc-65398 [Santa Cruz Biotechnology, Santa Cruz]; anti-Yes-associated protein [YAP], 1:100, sc-101199 [Santa Cruz]; antipalladin, 1:100, sc-166563 [Santa Cruz]), followed by three washes with PBS. Samples were incubated with secondary antibodies (rabbit anti-goat IgM, 1:100, bs0370R-FITC [Bioss Antibodies]; mouse anti-rabbit IgM, 1:100, bs-0369M-FITC [Bioss Antibodies]; anti-mouse IgG Fab2 Alexa Fluor 488, 1:200, \#4408S [Cell Signaling Technology]) and rhodamine phalloidin (1:50, R415; Invitrogen) for $2 \mathrm{~h}$ at $37^{\circ} \mathrm{C}$. Finally, nucleic acid staining 
was performed by incubating the cells with DAPI (D9542; Sigma-Aldrich) for $5 \mathrm{~min}$.

Samples were mounted on microscope slides and imaged by fluorescence microscopy (Eclipse Ti-S, Nikon) equipped with a confocal system (UltraVIEW VoX, PerkinElmer). Images were acquired at 405,488 , and $560 \mathrm{~nm}$ excitation under the same conditions (exposure time, intensity, and sensitivity). The fluorescent signal was quantified using Volocity. Nuclear YAP or palladin intensity was determined according to the region confined by DAPI staining. Cytosolic YAP intensity was determined by the region confined by actin staining and without the region delimited by DAPI staining. For quantification of YAP nuclear localization, the ratio of YAP distribution between the cytoplasm and nucleus was analyzed in 35-70 cells for each experimental condition.

\section{Atomic force microscopy (AFM)}

CCD-1095Sk cells were cultured on substrates with different stiffnesses for at least three days before AFM measurements. To assess the elastic modulus of the cells, we used an AFM instrument (5500; Keysight) combined with an inverted microscope (TE2000U; Nikon). Each cell was probed to record the approach part of the forcedistance curves at the cell nucleus region at a frequency of $1 \mathrm{~Hz}$. Silica spheres with a diameter of approximately $18 \mu \mathrm{m}$ were attached to tipless AFM cantilevers (TLCONT; Nanosensor) based on our previous method [22]. The force-distance curves were converted into force-indentation curves and fitted to the spherical Hertz model to calculate the Young's modulus of the CCD-1095Sk cells [23]. Before indention tests, the spring constant $(K$, $0.18 \mathrm{~N} \mathrm{~m}^{-1}$ ) of the cantilever was determined using the thermal tune method. The deflection sensitivity $(S$, $35.90 \mathrm{~nm} \mathrm{~V}^{-1}$ ) of the cantilever was measured in fluid according to the slope of the force-distance curves acquired on the glass substrate.

\section{Analysis of human cytokine antibody array}

Soluble cytokines in the prepared preconditioned medium (PCM) were detected using a Human Cytokine Array G5 (AAH-CYT-G5; RayBio), in which 80 human cytokines were included (Supplemental Table S1). The expression of each cytokine was determined by a fluorescence laser scanner. For each group without inhibitors, three biological replicates were used; only one replication assay was performed in PCM prepared with blebbistatin on a substrate with a stiffness of $46.7 \mathrm{kPa}$. According to the manufacturer's instructions, only average values of more than 50 were considered valid values.
Differences with more than 2-fold change or $p$ values of less than 0.05 were considered significant.

\section{Signaling manipulations}

The nonmuscle myosin inhibitor blebbistatin (B0560; Sigma-Aldrich) and Rho-associated protein kinase (ROCK) inhibitor Y27632 (ab120129; Abcam) were used at 25 and $10 \mu \mathrm{mol} \mathrm{L}{ }^{-1}$, respectively. Prior to fluorescent imaging, cytokine detection, or AFM measurements, inhibitors were incubated with CCD-1095Sk cells for $48 \mathrm{~h}$.

\section{Statistical analysis}

Data with homogeneous variances and following the normal distribution were expressed as the mean \pm standard deviation, and a parametric statistical method (oneway ANOVA, Student-Newman-Keuls, or Student's $t$ tests) was used to examine differences. For groups that did not follow the normal distribution or demonstrate nonhomogeneous variance, the median value was used to represent the data, and a nonparametric statistical method (Kruskal-Wallis test with NPAR1WAY procedure) was applied for statistical analysis. A $p$ value $<0.05$ was considered statistically significant. For each statistical analysis, at least three replicates were included, and the analysis was performed with SAS 9.0.

\section{RESULTS}

\section{Substrate stiffness modulated the effect of CAFs on tumor cell proliferation}

To investigate how stiffness heterogeneity of the tumor stroma induces the double-edged sword effect of CAFs on anticancer therapy, we first identified CAF markers in CCD-1095Sk human breast fibroblasts established from normal breast skin of a patient who had infiltrating ductal carcinoma. We found positive expression of CAF markers, such as fibroblast-activating protein (FAP; Fig. 1a), $\alpha$-smooth muscle actin ( $\alpha$-SMA; Fig. 1b), and palladin (Supplemental Fig. S1) in CCD-1095Sk cells. In addition, cytokines and chemokines, such as interleukin (IL)-6, IL8, IL-10, tumor necrosis factor (TNF), transforming growth factor (TGF)- $\beta$, and regulated upon activation, normal $\mathrm{T}$ cell expressed and secreted (RANTES), which are known to be secreted by CAFs, were also detected in the PCM of CCD-1095Sk cells (Table S1) [6]. Taken together, our results suggest that CCD-1095Sk exhibited the CAFs characteristics.

The stiffness of breast cancer tissue is increased by one order of magnitude compared with that of normal breast 

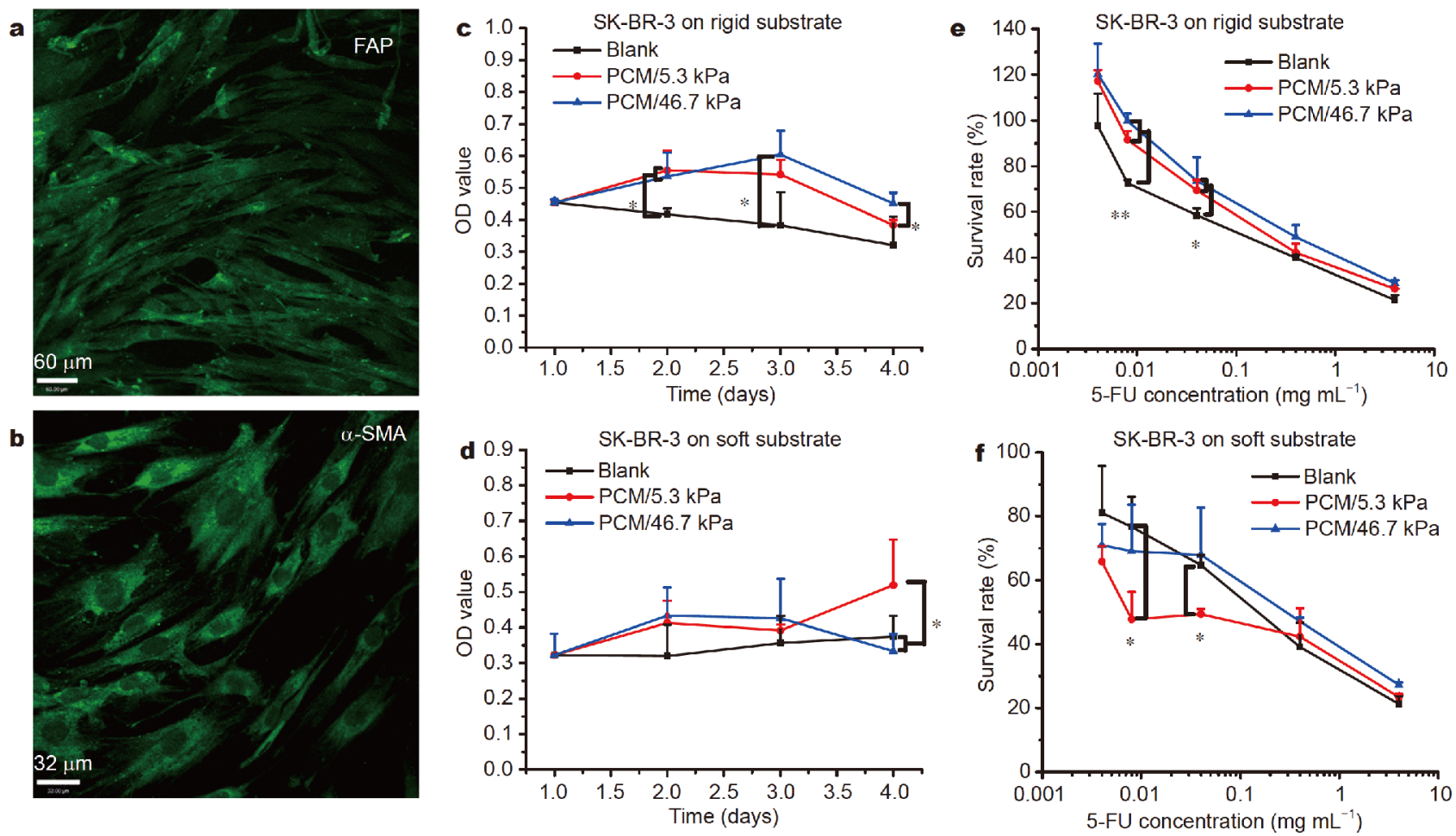

Figure 1 Proliferation and survival of SK-BR-3 cells in response to PCM from CCD-1095Sk cells (CAFs). (a) Fluorescent imaging of fibroblastactivating protein (FAP) expressed in CCD-1095Sk cells. (b) Fluorescent imaging of $\alpha$-SMA expressed in CCD-1095Sk cells. (c) Proliferation of SKBR-3 cells on rigid substrate (plastic) in response to PCM prepared on different substrates. (d) Proliferation of SK-BR-3 cells on soft substrate $(5.3 \mathrm{kPa})$ in response to PCM prepared on different substrates. (e) SK-BR-3 cell survival rates in response to PCM prepared on different substrates at different 5-fluorouracil (5-FU) concentrations when grown on rigid substrate (plastic). (f) SK-BR-3 survival rates in response to PCM prepared on different substrates at different 5 -FU concentrations when grown on soft substrate $(5.3 \mathrm{kPa})$. Statistical analysis was performed using one-way ANOVA with Student-Newman-Keuls Test. ${ }^{*} p<0.05,{ }^{* *} p<0.01$.

tissue [24]. PDMS with stiffnesses of $5.3 \pm 0.2$ and $46.7 \pm 2.1 \mathrm{kPa}$, both of which are physiologically relevant, was applied to mimic the heterogeneous stiffness of the mammary tumor stroma [3,14]. These PDMS stiffness measurements were determined using an ElectroForce 3100 , as described previously [14]. We then investigated the effects of PCM prepared from CCD-1095Sk cells growing on substrates of different stiffnesses (5.3 and $46.7 \mathrm{kPa}$, abbreviated as $\mathrm{PCM} / 5.3 \mathrm{kPa}$ and $\mathrm{PCM} / 46.7 \mathrm{kPa}$, respectively) on the proliferation of SK-BR-3 breast cancer cells. Our results showed that $\mathrm{PCM} / 46.7 \mathrm{kPa}$ increased cell proliferation after four days relative to that of $\mathrm{PCM} / 5.3 \mathrm{kPa}$ on the rigid substrate (plastic) $(F=8.45, p<$ 0.05; Fig. 1c). However, on soft substrate $(5.3 \mathrm{kPa})$, tumor cell proliferation was increased by $\mathrm{PCM} / 5.3 \mathrm{kPa}$ compared with that of cells exposed to $\mathrm{PCM} / 46.7 \mathrm{kPa}$ and control medium ( $F=9.79, p<0.05$; Fig. $1 \mathrm{~d})$. Hence, our results suggested that the effects of CAFs on tumor cell proliferation appeared to be modulated by the stiffness of the substrate on which CAFs and tumor cells grew.

\section{Diverse effects of PCM and substrate stiffness on tumor} cell responses to the chemotherapeutic drug 5-FU

We then studied whether the PCM prepared on different substrate stiffness also influenced the responses of SKBR-3 cells to 5-FU, which is widely used to treat breast cancer owing to its ability to halt cell division by interfering with the synthesis of DNA and RNA [25]. When SK-BR-3 cells were cultured on rigid plastic substrates, the survival rates of SK-BR-3 cells incubated with PCM were significantly increased at 5-FU concentrations of 0.008 and $0.04 \mathrm{mg} \mathrm{mL}^{-1}$ compared with that in the blank (no PCM) group (Fig. 1e). $\mathrm{IC}_{50}$ values were calculated to evaluate the effects of PCM. The results showed that the $\mathrm{IC}_{50}$ value in the blank group $\left(0.11 \pm 0.04 \mathrm{mg} \mathrm{m}^{-1}\right)$ was significantly lower than those in PCM-treated groups $\left(0.33 \pm 0.08 \mathrm{mg} \mathrm{mL}^{-1}\right.$ for $\mathrm{PCM} / 46.7 \mathrm{kPa}$ and $0.30 \pm$ $0.05 \mathrm{mg} \mathrm{mL}^{-1}$ for PCM/5.3 kPa; one-way analysis of variance [ANOVA], $F=11.56, p<0.05$; Fig. S2a). However, when tumor cells were cultured on the soft PDMS substrate (5.3 kPa; Fig. 1f), the survival rates of SK-BR-3 cells 

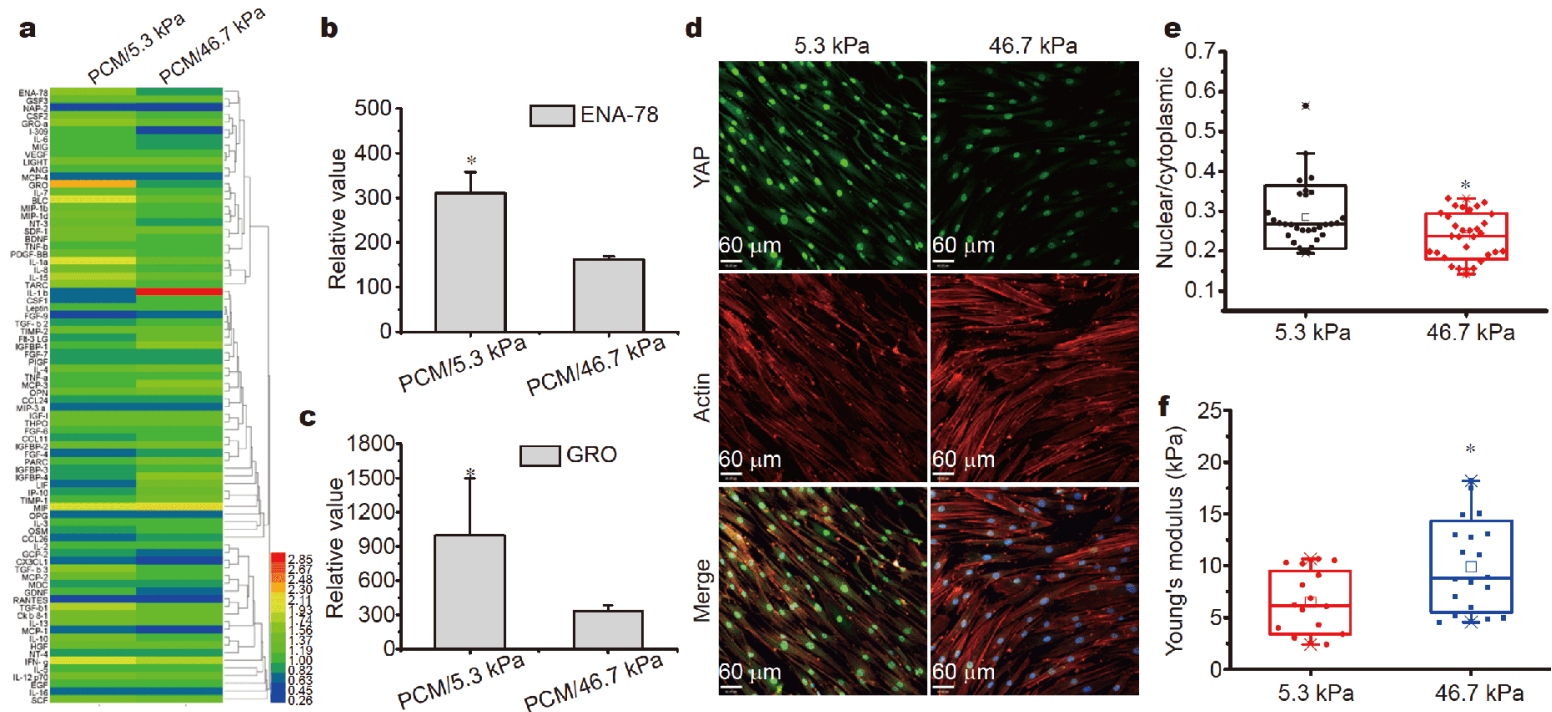

Figure 2 Expression analysis of cytokines, Yes-associated protein (YAP), and stress fibers in CCD-1095Sk cells (CAFs) grown on substrates with stiffnesses of 5.3 and $46.7 \mathrm{kPa}$. (a) Heat map of cytokine secretion from CCD-1095Sk cells cultured on 5.3 and $46.7 \mathrm{kPa}$. (b) Relative expression of GRO in PCM/5.3 kPa and PCM/46.7 kPa groups. (c) Relative expression of epithelial-derived neutrophil-activating peptide 78 (ENA-78) in PCM/ $5.3 \mathrm{kPa}$ and $\mathrm{PCM} / 46.7 \mathrm{kPa}$ groups. (d) Fluorescent characterization of YAP and stress fibers in CCD-1095Sk cells grown on substrates with stiffnesses of 5.3 and $46.7 \mathrm{kPa}$. (e) Quantification of YAP nuclear distribution in CCD-1095Sk cells with nuclear/cytoplasmic values grown on substrates with stiffnesses of 5.3 and $46.7 \mathrm{kPa}$. (f) Stiffness characterization of CCD-1095Sk cells grown on substrates with stiffnesses of 5.3 and $46.7 \mathrm{kPa}$ employing atomic force microscopy. For (b) and (c), statistical analysis was performed using Student's $t$-tests. For (e) and (f), statistical analysis was performed using Kruskal-Wallis tests with the NPAR1WAY procedure in SAS. ${ }^{*} p<0.05$.

were significantly reduced by PCM compared with that in the blank (no PCM) group at 5-FU concentrations of 0.008 and $0.04 \mathrm{mg} \mathrm{mL}^{-1}$. Interestingly, no obvious differences were found between the blank group and PCM/ $46.7 \mathrm{kPa}$ group. One-way ANOVA of $\mathrm{IC}_{50}$ values also indicated that the values in the blank $(0.14 \pm$ $\left.0.05 \mathrm{mg} \mathrm{mL}^{-1}\right)$ and $\mathrm{PCM} / 46.7 \mathrm{kPa}$ groups $(0.23 \pm$ $0.05 \mathrm{mg} \mathrm{mL}^{-1}$ ) were significantly higher than that in the $\mathrm{PCM} / 5.3 \mathrm{kPa}$ group $\left(0.03 \pm 0.01 \mathrm{mg} \mathrm{mL}^{-1} ; F=17.99, p<\right.$ 0.01; Fig. S2b). Thus, CAFs appeared to have diverse effects on tumor cell responses toward cytotoxicity drugs, and such associations may be affected by the substrate stiffness. That is, when tumor cells were grown on the stiff substrate, the cytotoxic effects of 5-FU were inhibited by PCM, regardless what level of substrate stiffness of CAFs were grown on; however, when tumor cells were cultured on the soft PDMS (5.3 $\mathrm{kPa})$ substrate, PCM prepared from the soft substrate $(5.3 \mathrm{kPa})$ actually enhanced the response of cancer cells to 5-FU. According to these results, stiffness heterogeneity promoted the role of CAFs as a double-edged sword in 5-FU therapy.

\section{Substrate stiffness induced cytokine secretion from CCD- 1095Sk cells}

In order to elucidate how stiffness influenced the effects of
CCD-1095Sk cells, we performed cytokine array analysis of the PCM from different groups. We hypothesized that the PCM-mediated effects may be associated with soluble factors secreted by CAFs [6]. To detect soluble factors secreted by CCD-1095Sk cells, we carried out an antibody-based cytokine array analysis of 80 cytokines with PCM prepared on different substrates. All groups displayed abundant expression of soluble factors, such as TGF- $\beta$, vascular endothelial growth factor (VEGF), RANTES, IL-6, epithelial-derived neutrophil-activating peptide 78 (ENA-78), growth-related oncogene (GRO), monocyte chemotactic protein (MCP)-1, stromal cell factor (SCF), macrophage migration inhibitory factor (MIF), and tissue inhibitor of metalloproteinases (TIMP; Fig. 2a and Table S1 online), factors usually associated with cell proliferation and drug responses. The expression levels of GRO and ENA-78 were significantly increased in the $\mathrm{PCM} / 5.3 \mathrm{kPa}$ group compared with those in the PCM/ $46.7 \mathrm{kPa}$ group (Fig. $2 \mathrm{~b}$ and c). Except for GRO and ENA78 , no obvious changes in other cytokines were identified between the PCM/5.3 kPa and PCM/46.7 kPa groups.

\section{Substrate stiffness altered the nuclear distribution of YAP in CCD-1095Sk cells}

We then investigated which pathways or targets in CCD- 
1095Sk cells were associated with substrate stiffness and differential cytokine expression. YAP has been reported to act as a nuclear relay for mechanical signals originating from the rigidity of the extracellular matrix, which is mediated by Rho GTPase activity and tension of the actomyosin cytoskeleton [26]. YAP nuclear accumulation activates the expression of GRO and ENA-78 through binding with the promoter of target genes as a transcription factor $[27,28]$. Hence, we next investigated whether YAP distribution in the nucleus was regulated by stiffness and resulted in differential GRO and ENA-78 expression. Employing confocal fluorescent microscopy, we observed that CCD-1095Sk cells grown on a stiffness of $5.3 \mathrm{kPa}$ showed elevated nuclear distribution compared with cells grown on a stiffness of $46.7 \mathrm{kPa}$ (Fig. 2d). Moreover, quantitative analysis of YAP localization also showed that the nuclear/cytoplasmic ratio of cells grown on a substrate with $5.3 \mathrm{kPa}$ stiffness was significantly higher than that of cells grown on a substrate with $46.7 \mathrm{kPa}$ stiffness $\left(\chi^{2}=5.97, p<0.05\right.$; Fig. 2e). The median (minimum-maximum) nuclear/cytoplasmic ratios of YAP in the 5.3 and $46.7 \mathrm{kPa}$ groups were $0.27(0.19-0.56)$ and 0.24 (0.14-0.33), respectively.

Because stress fibers play critical roles in the translocation of YAP from the cytoplasm to the nucleus, we next analyzed the stiffnesses of CCD-1095Sk cells and the stress fibers of these cells in relation to the substrate stiffness. Cells on a stiff substrate showed prominent stress fibers throughout the cytoplasm; in contrast, cells on a soft substrate $(5.3 \mathrm{kPa})$ only formed dispersed stress fibers at the outer edge (Fig. 2d). AFM measurements demonstrated that the Young's modulus of CCD-1095Sk cells grown on a stiffness of $5.3 \mathrm{kPa}$ was less than that of cells grown on a stiffness of $46.7 \mathrm{kPa}\left(\chi^{2}=6.21, p<0.05\right.$; Fig. 2f). The median values (minimum-maximum) of Young's modulus in the 5.3 and $46.7 \mathrm{kPa}$ groups were $6.13(2.40-10.66) \mathrm{kPa}$ and $8.79(4.55-18.19) \mathrm{kPa}$, respectively. These results suggested that stress fiber formation and stiffness of CCD-1095Sk cells were reduced on softer substrates, which may explain the increased YAP nuclear localization in cells grown on the soft substrate compared with that of cells grown on the stiff substrate.

\section{Stress fiber inhibitors modified the effects of CAFs on the survival of SK-BR-3 cells grown on the PDMS substrate}

To further define the roles of stress fibers in the mechanotransduction of CAFs, stress fiber formation was inhibited by the nonmuscle myosin inhibitor blebbistatin $\left(25 \mu \mathrm{mol} \mathrm{L}^{-1}\right)$ or ROCK inhibitor Y27632 $\left(10 \mu \mathrm{mol} \mathrm{L}^{-1}\right)$ [29]. Cytokine array analysis showed that the expression of soluble cytokines in the PCM $/ 46.7 \mathrm{kPa}$ group was downregulated after blebbistatin treatment (Fig. 3a and Table S2). Most downregulated cytokines were involved in the progression and growth of tumors. In particular, the expression levels of GRO and ENA-78 were decreased by 3.78 - and 3.03-fold, respectively (Fig. 3b). Our results suggested that the secretion of soluble factors was regulated by stress fiber structures, which could be modulated by substrate stiffness.

We further investigated the relationships of stress fiber formation, substrate stiffness, PCM of CAFs, and cancer cell survival in response to 5-FU. Before the experiment, we confirmed that blebbistatin and Y27632 did not have cytotoxic effects on cancer cells grown on PDMS with a stiffness of $5.3 \mathrm{kPa}$, with or without PCM (Fig. S3). SKBR-3 cells exposed to different 5-FU concentrations were incubated with PCMs prepared from CCD-1095SSK cells treated with either blebbistatin or Y27632 on stiffnesses of 5.3 and $46.7 \mathrm{kPa}$. When SK-BR-3 cells were grown on PDMS with a stiffness of $5.3 \mathrm{kPa}$, the difference in survival between $\mathrm{PCM} / 5.3 \mathrm{kPa}$ and $\mathrm{PCM} / 46.7 \mathrm{kPa}$ groups was abrogated in the presence of blebbistatin (Fig. 3c) or Y27632 (Fig. 3d). These results indicated that stress fiber formation of CAFs played an essential role in regulating the responses of cancer cells to 5-FU.

\section{Stress fiber inhibitors reduced the nuclear distribution of YAP in CCD-1095Sk cells}

Because YAP nuclear translocation was mediated by stress fibers, we further investigated whether stress fiber inhibitors changed the YAP nuclear distribution of CCD1095Sk cells. We found that YAP nuclear distributions in CCD-1095Sk cells on a less-stiff substrate $(5.3 \mathrm{kPa})$ and stiffer substrate $(46.7 \mathrm{kPa})$ were both reduced after blebbistatin or Y27632 treatment (Fig. S4). Furthermore, based on the confocal imaging, no obvious differences in YAP expression and nuclear distribution were observed between CCD-1095Sk cells grown on substrates with 5.3 and $46.7 \mathrm{kPa}$ stiffness after blebbistatin or Y27632 treatment (Fig. 4a). Quantitative YAP fluorescence intensity in the nucleus versus cytoplasm showed no significant differences in nuclear/cytoplasmic ratios between CCD1095Sk cells grown on substrates with 5.3 or $46.7 \mathrm{kPa}$ stiffness upon blebbistatin treatment $\left(\chi^{2}=0.01, p=0.93\right.$, Fig. $4 c, i)$ or Y27632 treatment $\left(\chi^{2}=0.15, p=0.70\right.$, Fig. $4 c$, ii). After blebbistatin and Y27632 treatment, the median (minimum-maximum) nuclear/cytoplasmic ratios of YAP in CCD-1095Sk cells grown on the substrate with $5.3 \mathrm{kPa}$ stiffness were $0.19(0.10-0.27)$ and $0.18(0.10-$ 0.28 ), respectively, whereas those of cells grown on the 


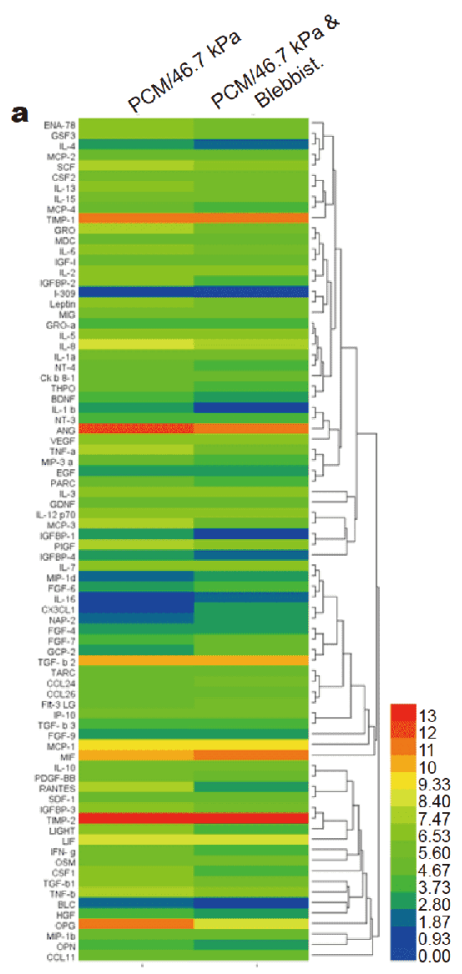

b

\begin{tabular}{|c|c|}
\hline Cytokines & Fold inhibition \\
\hline RANTES & 45.05 \\
\hline CSF1 & 6.82 \\
\hline IL-1 b & 6.53 \\
\hline IGFBP-2 & 6.12 \\
\hline IGFBP-1 & 5.90 \\
\hline MCP-3 & 5.85 \\
\hline OPG & 4.75 \\
\hline LIGHT & 4.17 \\
\hline GRO & 3.78 \\
\hline IFN-g & 3.26 \\
\hline ENA-78 & 3.03 \\
\hline TNF-a & 2.83 \\
\hline HGF & 2.50 \\
\hline I-309 & 2.38 \\
\hline PDGF-BB & 2.28 \\
\hline IGFBP-4 & 2.10 \\
\hline IL-4 & 2.10 \\
\hline ANG & 2.00 \\
\hline
\end{tabular}
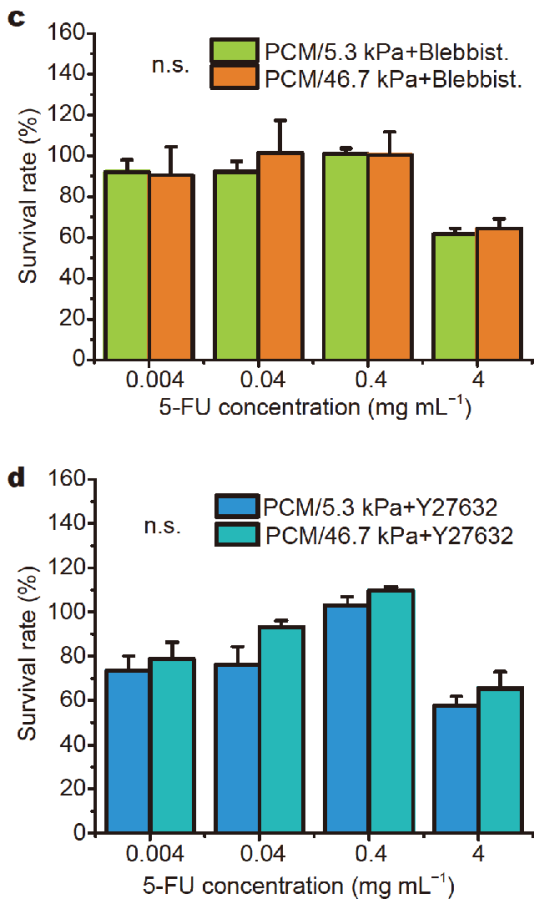

Figure 3 Influence of stress fiber inhibitors on cytokine secretion from CCD-1095Sk cells (CAFs) and survival of SK-BR-3 cells. (a) Heat map of cytokine secretion from CCD-1095Sk cells before and after blebbistatin (blebbist.) inhibition. (b) List of cytokines inhibited by more than 2-fold in (a). (c) Cancer cell survival rates in cells grown in PCM from CCD-1095Sk cells prepared on substrates with stiffnesses of 5.3 and $46.7 \mathrm{kPa}$ after treatment with blebbist. (d) Cancer cell survival rates in cells grown in PCM from CCD-1095Sk cells prepared on substrates with stiffnesses of 5.3 and $46.7 \mathrm{kPa}$ after treatment with Y27632. Statistical analysis was performed using pooled two sample $t$-tests. n.s., not significant.

substrate with $46.7 \mathrm{kPa}$ stiffness were $0.20(0.10-0.26)$ and 0.19 (0.08-0.41), respectively. Hence, these results showed that stress fiber inhibitors abrogated alterations in YAP nuclear distribution resulting from differences in the substrate stiffness on which CCD-1095Sk cells were grown.

The structures of stress fiber upstream of YAP translocation were also affected by the inhibitors. For example, organized stress fibers were significantly decreased in CCD-1095Sk cells grown on stiffnesses of 5.3 and $46.7 \mathrm{kPa}$ after treatment with blebbistatin or Y27632 (Fig. 4b). Consistent with these results, stiffness differences in CCD-1095Sk cells grown on substrates with stiffnesses of 5.3 and $46.7 \mathrm{kPa}$ were also abolished upon treatment with blebbistatin $\left(\chi^{2}=0.03, p=0.85\right.$, Fig. $4 c$, iii) or Y27632 $\left(\chi^{2}=0.44, p=0.51\right.$, Fig. $4 c$, iv $)$. After blebbistatin and Y27632 treatment, the median values (minimum-maximum) for Young's modulus of CCD1095Sk cells grown on the substrate with $5.3 \mathrm{kPa}$ stiffness were $6.80(2.51-12.13)$ and $6.25(2.57-15.82) \mathrm{kPa}$, respectively, whereas those of cells grown on the substrate with $46.7 \mathrm{kPa}$ stiffness were $7.48(2.84-11.22)$ and 5.90 $(2.66-14.72) \mathrm{kPa}$, respectively. We further found that the stiffness of CCD-1095Sk cells grown on the substrate with $46.7 \mathrm{kPa}$ stiffness was significantly decreased by the inhibitors, whereas that of cells grown on the substrate with $5.3 \mathrm{kPa}$ stiffness did not change (Fig. S5).

\section{DISCUSSION}

The tumor microenvironment demonstrates a heterogeneous distribution of both its biological/biochemical constitutes and biomechanical properties, which play critical roles in regulating tumor cell phenotypes, including proliferation, invasion, metastasis, and treatment response [3,4]. Nonmalignant stromal cells, such as fibroblasts, immune cells, vascular cells, and even neurons in the tumor microenvironment, have diverse biochemical effects that can be either beneficial or detrimental [30-32]. The mechanisms for such a divergent, often opposite, effect are unclear. Here, for the first time, we found that stroma substrates played important roles in inducing CAFs as a double-edged sword in cancer cell 

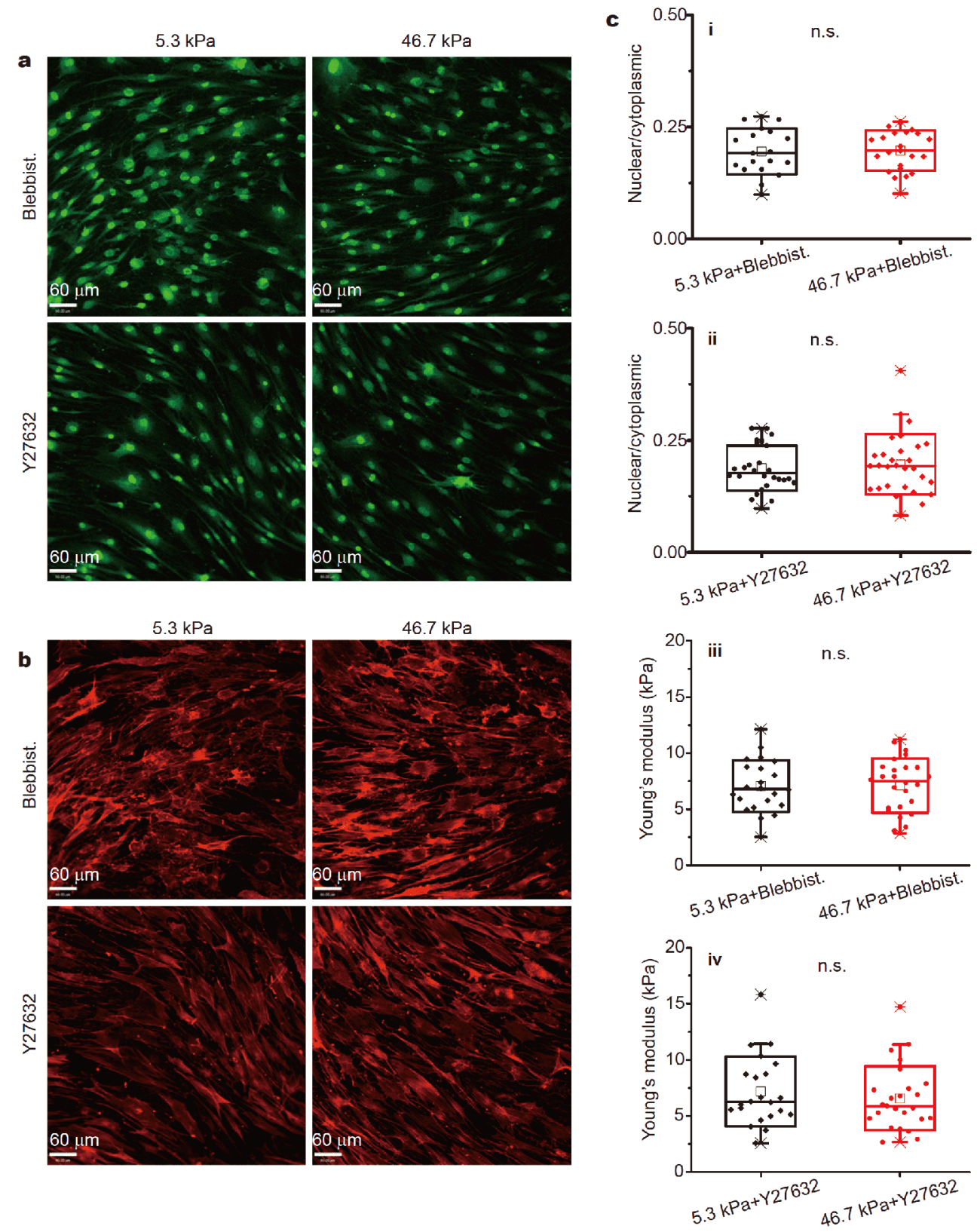

Figure 4 Influence of stress fiber inhibitors on YAP nuclear localization and stress fiber formation in CCD-1095Sk cells (CAFs) grown on substrates with stiffnesses of 5.3 and $46.7 \mathrm{kPa}$. (a) Fluorescent imaging of YAP distribution in CCD-1095Sk cells after inhibition with blebbistatin or Y27632 for cells grown on substrates with stiffnesses of 5.3 and $46.7 \mathrm{kPa}$. (b) Fluorescent imaging of stress fibers in CCD-1095Sk cells after inhibition with blebbistatin or Y27632 for cells grown on substrates with stiffnesses of 5.3 and $46.7 \mathrm{kPa}$. (c) Quantification of YAP distribution in CCD-1095Sk cells grown on substrates with stiffnesses of 5.3 and $46.7 \mathrm{kPa}$ after inhibition with blebbistatin (i) or Y27632 (ii); stiffness characterization of CCD-1095Sk cells grown on substrates with stiffnesses of 5.3 and $46.7 \mathrm{kPa}$ after inhibition with blebbistatin (iii) or Y27632 (iv). Statistical analysis was performed using Kruskal-Wallis tests with the NPAR1WAY procedure in SAS. n.s., not significant.

proliferation and drug response. The complex interplay and crosstalk between stroma biomechanical properties, such as stiffness, and CAF-mediated chemo- and mechanotransduction together regulated cell proliferation and drug sensitivity. The CAF-mediated process involved both cytokine secretion and stress fiber formation.

The roles of CAFs and stroma stiffness in tumor growth have been widely investigated. Previous in vitro studies have shown that CAFs enhance tumor cell survival in response to both chemotherapy and targeted therapy when 
cocultured on plastic systems [33-35]. Stromal fibroblasts of pancreatic ductal adenocarcinoma tissues in mice promote the suppression of tumor growth upon ganciclovir administration [20,21]. Additionally, Liu et al. [2] found that biophysical cues in vivo, specifically matrix stiffness, were potential targets of mesenchymal stem cell-based vectors for cancer treatment, whereas manipulating stroma stiffness may affect the outcomes of cancer treatment. Similarly, we found that when tumor cells were grown on soft PDMS substrates (5.3 kPa), PCM also prepared on the $5.3 \mathrm{kPa}$ substrate led to inhibition of tumor cell survival in response to 5-FU, whereas PCM prepared on a stiffer substrate $(46.7 \mathrm{kPa})$ had no obvious effect.

The widely investigated antitumor agents include chemo drugs, targeted drugs, and recently developed nanoparticles, such as nanorods and nanocrystals, which are used for photothermal therapy [36,37]. Cytokine crosstalk between tumor cells and CAFs is responsible for drug resistance [38]. In CCD-1095Sk cell supernatants, we detected abundant expression of cytokines, such as TGF$\beta$, VEGF, RANTES, IL-6, ENA-78, GRO, MCP-1, SCF, TNF, angiotensin, osteoprotegerin, leukemia inhibitory factor, MIF, and TIMP. TGF- $\beta$ on a stiff substrate has been reported to be a critical mediator of fibroblast/tumor crosstalk, modulating 5-FU resistance via activation of the epithelial-mesenchymal transition (EMT) process or protection of breast cancer cells from DNA damageinduced apoptosis via inhibition of p53 [38,39]. Moreover, studies have shown that the chemotherapy resistance conferred by TGF- $\beta$ is abrogated by decreasing stiffness, which inhibits the TGF- $\beta$-induced EMT $[40,41]$. Because no significant differences in TGF- $\beta$ secretion were detected between $\mathrm{PCM} / 5.3 \mathrm{kPa}$ and $\mathrm{PCM} / 46.7 \mathrm{kPa}$ groups (Fig. S6), we examined other mechanisms, specifically cytokines such as GRO and ENA-78, which showed elevated secretion in the $\mathrm{PCM} / 5.3 \mathrm{kPa}$ group. Indeed, on soft PDMS substrate, GRO and ENA-78 promoted cell cycle progression in tumor cells and thus increased sensitivity to 5 -FU $[42,43]$. The secretion of GRO and ENA-78 is regulated by nuclear localization of YAP, a mechanosensitive transcriptional co-activator that plays various roles in tissue homeostasis, organ development, and oncogenic transformation [44]. YAP nuclear accumulation in fibroblasts is known to be positively correlated with substrate stiffness, which can vary from less than $500 \mathrm{~Pa}$ to more than $10 \mathrm{kPa}[29,45]$. Another study further confirmed that apart from substrate stiffness, molecular clutches also play critical roles in YAP translocation, which is promoted on intermediate-stiffness substrates and collapsed on high-stiffness substrates by increasing the ligand spacing [46]. Consistent with the previous findings, we demonstrated that collagen-coated PDMS with a stiffness of $5.3 \mathrm{kPa}$ promoted more YAP nuclear accumulation compared with PDMS with a stiffness of $46.7 \mathrm{kPa}$, which accounted for the increased expression of GRO and ENA-78 in the PCM $/ 5.3 \mathrm{kPa}$ group. We also find that YAP nuclear translocation was inhibited by the stress fiber inhibitors, suggesting that YAP functioned downstream of stress fiber in mechanotransduction of stiffness.

Despite obvious limitations, such as the in vitro nature of the study, and the use of a single cell line/single chemotherapeutic drug, our study still provided strong evidence that the complex interplay among tumor cells, stromal cells (CAFs), and mechanical factors in the tumor microenvironment contributed to the observed malignant phenotypes, including tumor proliferation and drug response. Fig. 5 demonstrates the double-edged sword behaviors of CAFs. We hypothesize that CAFs sense the stiffness of the extracellular matrix by mechanotransduction of the integrin complex, which couples with ROCK to deliver mechanical signals to stress fibers. Stress fibers transmit extracellular mechanical signals and mediate YAP nuclear translocation through Hippo signaling. In the nucleus, YAP combines with the TEAD family of transcription factors and activates the secretion of cytokines, such as TGF- $\beta$, GRO, and ENA-78, which regulate the proliferation of cancer cells [47]. When tumor cells were grown on the rigid substrate, TGF- $\beta$ promoted tumor survival; in contrast, when tumor cells and CAFs were both grown on the soft substrate $(5.3 \mathrm{kPa})$, nuclear YAP inhibited cancer cell growth by upregulation of GRO and ENA-78 expression in CAFs. This process could be suppressed using inhibitors of nonmuscle myosin or ROCK, which disrupted the structures of stress fibers.

Tumors are known to exhibit heterogeneous features $[48,49]$. In fact, although the tumor stroma is often stiff, the tumor cells themselves are soft [50]. Stiffness heterogeneity influences cytokine crosstalk in tumor-stromal interfaces and modulates chemotherapy responses. Combined with our recent studies, these findings suggest that mechanical factors in the tumor microenvironment function not only via direct regulation of cell mechanotransduction $[14,15]$, but also by modulating tumorstromal cell interactions, which have critical implications for clinical cancer treatment and drug screening. Recent studies have focused on the tumor microenvironment for the development of pharmacological strategies to inhibit cancer because stromal cells are genetically stable and can 


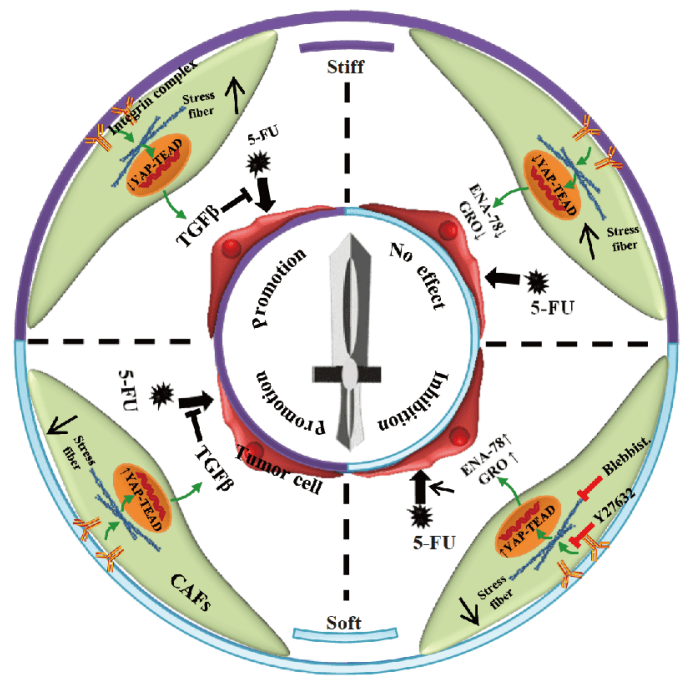

Figure 5 Illustration of the mechanism of the double-edged sword effect of CAFs on tumor drug responses. CAFs sense the stiffness of the extracellular matrix by mechanotransduction of the integrin complex, which delivers the mechanical signal to stress fibers. Stress fibers transmit extracellular mechanical signals and mediate YAP nuclear translocation, which then activates the secretion of cytokines from CAFs. When tumor cells were grown on the rigid substrate, TGF- $\beta$ promoted tumor survival. When tumor cells and CAFs were both grown on the soft substrate $(5.3 \mathrm{kPa})$, nuclear YAP inhibited cancer cell growth by upregulation of GRO and ENA-78 expression in CAFs. The process was suppressed by blebbistatin or Y27632, which disrupted the structures of stress fibers.

be re-educated for normalization [31]. Based on these new strategies, stromal cells and cytokines have been targeted by various agents, such as small molecules, antibodies, and nanoparticles [51,52]; however, satisfactory efficacy in the clinical setting has not been achieved, likely owing to the double-edged sword effect conferred by stiffness heterogeneity. Hence, integral regulation of the tumor mechanical microenvironment combined with chemotherapy or target therapy may be a better approach for cancer treatment.

\section{CONCLUSIONS}

In conclusion, stiffness heterogeneity induced the doubleedged sword behaviors of CAFs in anticancer therapy. In response to changes in the mechanical microenvironment, CAFs regulate their stiffness and mechanotransduction, such as stress fiber formation, nuclear YAP distribution, and cytokine secretion, which further influence tumor proliferation and chemotherapeutic efficacy. Thus, mechanical intervention of the tumor microenvironment should be an important component of the overall anticancer drug design and treatment, which can be regarded as a relevant biomechanopharmacology strategy $[53,54]$.

\section{Received 7 November 2018; accepted 13 December 2018;} published online 4 January 2019

1 Mitchell MJ, Jain RK, Langer R. Engineering and physical sciences in oncology: Challenges and opportunities. Nat Rev Cancer, 2017, 17: 659-675

2 Liu L, Zhang SX, Liao W, et al. Mechanoresponsive stem cells to target cancer metastases through biophysical cues. Sci Transl Med, 2017, 9: eaan2966

3 Plodinec M, Loparic M, Monnier CA, et al. The nanomechanical signature of breast cancer. Nat Nanotech, 2012, 7: 757-765

4 Acerbi I, Cassereau L, Dean I, et al. Human breast cancer invasion and aggression correlates with ECM stiffening and immune cell infiltration. Integr Biol, 2015, 7: 1120-1134

$5 \mathrm{Hu} \mathrm{N}$, Cao Y, Ao Z, et al. Flow behavior of liquid metal in the connected fascial space: Intervaginal space injection in the rat wrist and mice with tumor. Nano Res, 2017, 11: 2265-2276

6 Kalluri R. The biology and function of fibroblasts in cancer. Nat Rev Cancer, 2016, 16: 582-598

7 Kharaishvili G, Simkova D, Bouchalova K, et al. The role of cancerassociated fibroblasts, solid stress and other microenvironmental factors in tumor progression and therapy resistance. Cancer Cell Int, 2014, 14: 41

8 Kalli M, Papageorgis P, Gkretsi V, et al. Solid stress facilitates fibroblasts activation to promote pancreatic cancer cell migration. Ann Biomed Eng, 2018, 46: 657-669

9 Levental KR, Yu H, Kass L, et al. Matrix crosslinking forces tumor progression by enhancing integrin signaling. Cell, 2009, 139: 891906

10 Yaqoob U, Cao S, Shergill U, et al. Neuropilin-1 stimulates tumor growth by increasing fibronectin fibril assembly in the tumor microenvironment. Cancer Res, 2012, 72: 4047-4059

11 Schrader J, Gordon-Walker TT, Aucott RL, et al. Matrix stiffness modulates proliferation, chemotherapeutic response, and dormancy in hepatocellular carcinoma cells. Hepatology, 2011, 53: 1192-1205

12 Ulrich TA, de Juan Pardo EM, Kumar S. The mechanical rigidity of the extracellular matrix regulates the structure, motility, and proliferation of glioma cells. Cancer Res, 2009, 69: 4167-4174

13 Nune KC, Li S, Misra RDK. Advancements in three-dimensional titanium alloy mesh scaffolds fabricated by electron beam melting for biomedical devices: Mechanical and biological aspects. Sci China Mater, 2018, 61: 455-474

14 Feng J, Tang Y, Xu Y, et al. Substrate stiffness influences the outcome of antitumor drug screening in vitro. Clin Hemorheol Microcirc, 2013, 55: 121-131

15 Liu C, Li X, Hua W, et al. Porous matrix stiffness modulates response to targeted therapy in breast carcinoma. Small, 2016, 12 : $4675-4681$

16 Sun Y. Tumor microenvironment and cancer therapy resistance. Cancer Lett, 2016, 380: 205-215

17 Krishnan V, Schaar B, Tallapragada S, et al. Tumor associated macrophages in gynecologic cancers. Gynecol Oncol, 2018, 149: 205-213

18 Chen J, Lin L, Yan N, et al. Macrophages loaded CpG and GNRPEI for combination of tumor photothermal therapy and immunotherapy. Sci China Mater, 2018, 61: 1484-1494

19 Yamamura Y, Asai N, Enomoto A, et al. Akt-Girdin signaling in 
cancer-associated fibroblasts contributes to tumor progression. Cancer Res, 2015, 75: 813-823

20 Öhlund D, Elyada E, Tuveson D. Fibroblast heterogeneity in the cancer wound. J Exp Med, 2014, 211: 1503-1523

21 Özdemir BC, Pentcheva-Hoang T, Carstens JL, et al. Depletion of carcinoma-associated fibroblasts and fibrosis induces immunosuppression and accelerates pancreas cancer with reduced survival. Cancer Cell, 2014, 25: 719-734

22 Geng L, Feng J, Sun Q, et al. Nanomechanical clues from morphologically normal cervical squamous cells could improve cervical cancer screening. Nanoscale, 2015, 7: 15589-15593

23 Butt HJ, Cappella B, Kappl M. Force measurements with the atomic force microscope: Technique, interpretation and applications. Surf Sci Rep, 2005, 59: 1-152

24 Paszek MJ, Zahir N, Johnson KR, et al. Tensional homeostasis and the malignant phenotype. Cancer Cell, 2005, 8: 241-254

25 Farmer P, Bonnefoi H, Anderle P, et al. A stroma-related gene signature predicts resistance to neoadjuvant chemotherapy in breast cancer. Nat Med, 2009, 15: 68-74

26 Dupont S, Morsut L, Aragona M, et al. Role of YAP/TAZ in mechanotransduction. Nature, 2011, 474: 179-183

27 Sharif GM, Schmidt MO, Yi C, et al. Cell growth density modulates cancer cell vascular invasion via hippo pathway activity and CXCR2 signaling. Oncogene, 2015, 34: 5879-5889

28 Wang G, Lu X, Dey P, et al. Targeting YAP-dependent MDSC infiltration impairs tumor progression. Cancer Discov, 2016, 6: 8095

29 Calvo F, Ege N, Grande-Garcia A, et al. Mechanotransduction and YAP-dependent matrix remodelling is required for the generation and maintenance of cancer-associated fibroblasts. Nat Cell Biol, 2013, 15: 637-646

30 Cole SW, Nagaraja AS, Lutgendorf SK, et al. Sympathetic nervous system regulation of the tumour microenvironment. Nat Rev Cancer, 2015, 15: 563-572

31 Quail DF, Joyce JA. Microenvironmental regulation of tumor progression and metastasis. Nat Med, 2013, 19: 1423-1437

32 De Palma M, Biziato D, Petrova TV. Microenvironmental regulation of tumour angiogenesis. Nat Rev Cancer, 2017, 17: 457-474

33 Straussman R, Morikawa $\mathrm{T}$, Shee $\mathrm{K}$, et al. Tumour micro-environment elicits innate resistance to RAF inhibitors through HGF secretion. Nature, 2012, 487: 500-504

34 Wilson TR, Fridlyand J, Yan Y, et al. Widespread potential for growth-factor-driven resistance to anticancer kinase inhibitors. Nature, 2012, 487: 505-509

35 Holohan C, Van Schaeybroeck S, Longley DB, et al. Cancer drug resistance: An evolving paradigm. Nat Rev Cancer, 2013, 13: 714726

36 Wu Y, Wang D, Li Y. Understanding of the major reactions in solution synthesis of functional nanomaterials. Sci China Mater, 2016, 59: 938-996

$37 \mathrm{Xu} \mathrm{X,} \mathrm{Lu} \mathrm{Y,} \mathrm{Yang} \mathrm{Y,} \mathrm{et} \mathrm{al.} \mathrm{Tuning} \mathrm{the} \mathrm{growth} \mathrm{of} \mathrm{metal-organic}$ framework nanocrystals by using polyoxometalates as coordination modulators. Sci China Mater, 2015, 58: 370-377

38 Paraiso KHT, Smalley KSM. Fibroblast-mediated drug resistance in cancer. Biochem Pharmacol, 2013, 85: 1033-1041

39 Brunen D, Willems SM, Kellner U, et al. TGF- $\beta$ : An emerging player in drug resistance. Cell Cycle, 2013, 12: 2960-2968

40 Leight JL, Wozniak MA, Chen S, et al. Matrix rigidity regulates a switch between TGF- $\beta 1$-induced apoptosis and epithelial-me- senchymal transition. Mol Bio Cell, 2012, 23: 781-791

41 Liu Y, He K, $\mathrm{Hu} \mathrm{Y}$, et al. YAP modulates TGF- $\beta 1$-induced simultaneous apoptosis and EMT through upregulation of the EGF receptor. Sci Rep, 2017, 7: 45523

42 Kawamura M, Toiyama Y, Tanaka K, et al. CXCL5, a promoter of cell proliferation, migration and invasion, is a novel serum prognostic marker in patients with colorectal cancer. Eur J Cancer, 2012, 48: 2244-2251

43 Wang B, Hendricks DT, Wamunyokoli F, et al. A growth-related oncogene/CXC chemokine receptor 2 autocrine loop contributes to cellular proliferation in esophageal cancer. Cancer Res, 2006, 66: 3071-3077

44 Low BC, Pan CQ, Shivashankar GV, et al. YAP/TAZ as mechanosensors and mechanotransducers in regulating organ size and tumor growth. FEBS Lett, 2014, 588: 2663-2670

45 Liu F, Lagares D, Choi KM, et al. Mechanosignaling through YAP and TAZ drives fibroblast activation and fibrosis. Am J PhysiolLung Cellular Mol Physiol, 2015, 308: L344-L357

46 Oria R, Wiegand T, Escribano J, et al. Force loading explains spatial sensing of ligands by cells. Nature, 2017, 196: 219-224

47 Zhao B, Ye X, Yu J, et al. Tead mediates YAP-dependent gene induction and growth control. Genes Dev, 2008, 22: 1962-1971

48 Hasebe T. Tumor-stromal interactions in breast tumor progression-significance of histological heterogeneity of tumor-stromal fibroblasts. Expert Opin Therap Targets, 2013, 17: 449-460

49 Sharma S, Santiskulvong C, Bentolila LA, et al. Correlative nanomechanical profiling with super-resolution F-actin imaging reveals novel insights into mechanisms of cisplatin resistance in ovarian cancer cells. Nanomed-Nanotechnol Biol Med, 2012, 8: 757-766

50 Cross SE, Jin YS, Rao J, et al. Nanomechanical analysis of cells from cancer patients. Nat Nanotech, 2007, 2: 780-783

51 Adams JL, Smothers J, Srinivasan R, et al. Big opportunities for small molecules in immuno-oncology. Nat Rev Drug Discov, 2015, 14: 603-622

52 Schmid D, Park CG, Hartl CA, et al. T cell-targeting nanoparticles focus delivery of immunotherapy to improve antitumor immunity. Nat Commun, 2017, 8: 1747

$53 \mathrm{Tu}$ Y. The discovery of artemisinin (qinghaosu) and gifts from Chinese medicine. Nat Med, 2011, 17: 1217-1220

54 Liao F, Li M, Han D, et al. Biomechanopharmacology: A new borderline discipline. Trends Pharmacol Sci, 2006, 27: 287-289

Acknowledgements This work was financially supported by the Postdoctoral Science Foundation Program of Chinese Academy of Medical Sciences \& Peking Union Medical College, the National Natural Science Foundation of China (NSFC) (31470905), and National Institutes of Health/National Cancer Institute (NIH/NCI) Grant R21, CA208196.

Author contributions Rao J, Han D, He J, and Liao F designed the experiments. Feng J, Li X, Rao E, and Zhang Q conducted experiments and analyzed the data. Feng J, Sharma S, Rao E, Han D and Rao J wrote the manuscript. All authors contributed to the general discussion.

Conflict of interest The authors declare no competing financial interest.

Supplementary information Supplementary data associated with this article is available in the online version of the paper 

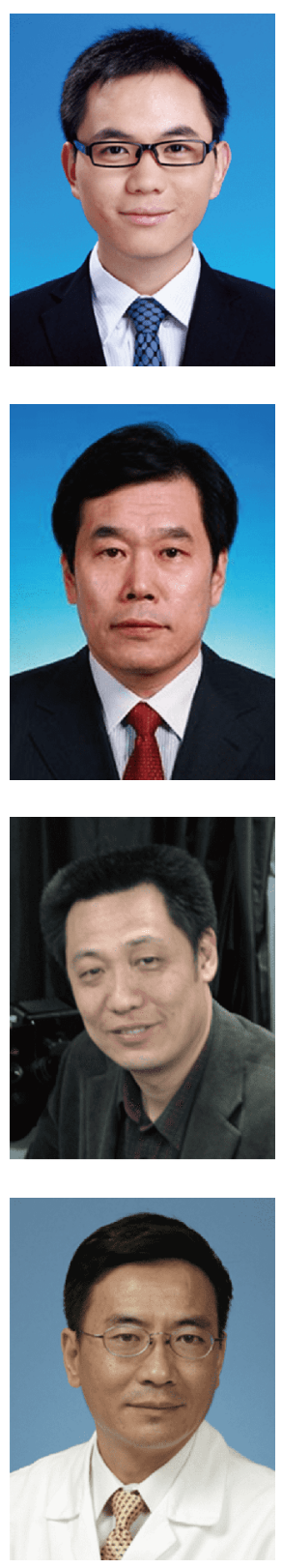

Jiantao Feng was born in 1987. He received his $\mathrm{PhD}$ degree in chemistry from the Department of Chemistry, Tsinghua University in 2014. He is currently doing postdoctoral research with Prof. Jie He and Prof. Jianyu Rao at Cancer Hospital, Chinese Academy of Medical Sciences. His research focuses on the biomechanopharmacology in tumor microenvironment.
Jie He is currently a professor in Cancer Hospital, Chinese Academy of Medical Sciences. He got his PhD degree in Peking Union Medical College in 1993. His research interest focuses on the surgical diagnosis and treatment of lung cancer and esophageal cancer.
Dong Han is currently a professor in the National Center for Nanoscience and Technology (NCNST). He received his clinical MSc degree (1998) and MD/PhD (2001) from China Academy of Chinese Medical Sciences. His research interest focuses on nanobiomedical imaging, functional biointerfaces and translational medicine.
Jianyu Rao is a tenured full professor and vice chair of the Department of Pathology and Laboratory Medicine at the University of California at Los Angeles (UCLA). He received his MD degree from Shanghai Medical University in 1984. His research focuses on cancer biomarker studies. He is also interested in developing new technologies for pathology especially cytopathology, including imaging and digital analysis, telepathology, nanotechnology, liquid biopsy, and AItechnology.

\section{肿瘤刚度异质性诱导的肿瘤相关成纤维细胞双刃剑行为研究}

冯建涛 ${ }^{1}$, Shivani Sharma ${ }^{2,3,4}$, Elizabeth $\mathrm{Rao}^{2}$, 李想 ${ }^{5}$ 张强 ${ }^{5}$, 廖福龙 ${ }^{5,6}$, 赫捷 ${ }^{1^{*}}$, 韩东 ${ }^{5^{*}}$, 饶建宇 ${ }^{2,3,4^{*}}$

摘要 肿瘤相关成纤维细胞(CAFs)在肿瘤的发展过程中发挥着双刃剑的作用, 但导致CAFs促进或者抑制肿瘤生存的关键因素还有待深入 研究. 本文中, 我们发现肿瘤细胞与CAFs所生长的基底的刚度异质性决定了上述两个相反的作用: 在化疗药物 5-氟尿嘧啶作用下, 当肿瘤 细胞培养在硬基底(塑料基底)上时, CAFs的条件培养液促进了肿瘤细胞的生存; 当肿瘤细胞与CAFs分别培养在软基底(5.3 kPa)上时, CAFs的条件培养液抑制了肿瘤细胞的生存. 基于原子力显微镜技术、激光共聚焦技术以及细胞因子芯片技术，我们发现CAFs中力学信 号传导下游的应力纤维分布以及YAP蛋白核迁移与CAFs的双刃剑行为密切相关. 因此调控肿瘤基质的力学传导有助于增强抗肿瘤药物 的疗效. 